Check for updates

Cite this: RSC Adv., 2018, 8, 17710

Received 26th February 2018

Accepted 1st May 2018

DOI: $10.1039 / \mathrm{c} 8 \mathrm{ra01679h}$

rsc.li/rsc-advances

\section{Co-delivery of doxorubicin and shRNA of Beclin1 by folate receptor targeted pullulan-based multifunctional nanomicelles for combinational cancer therapy}

\author{
Lili Chen, ${ }^{\text {ab }}$ Ming Qian, ${ }^{\mathrm{b}}$ Liuwei Zhang, ${ }^{\mathrm{b}}$ Jing Xia, ${ }^{\mathrm{b}}$ Yongming Bao, \\ Jingyun Wang, (D)*ab Lianying Guo ${ }^{c}$ and Yachen $\mathrm{Li}^{* \mathrm{C}}$
}

Doxorubicin (DOX) is a widely-used effective antitumor agent. However, its clinical application is limited due to its side effects including anti-apoptotic defense of cancer cells caused by DOX-induced autophagy and deleterious effects in normal tissues. Therefore, in this study, a new folate (FA)-decorated amphiphilic bifunctional pullulan-based copolymer (named as FPDP) was developed as an efficient nano-carrier for the co-delivery of DOX and short hairpin RNA of Beclin1, a pivotal autophage-related gene, to enhance the anticancer effect of DOX by the blockade of the Beclin1 protein mediated autophagy process. In FPDP molecules, pullulan was modified with lipophilic desoxycholic acid for the formation of micelles, the introduced low molecular weight (1 kDa) branched polyethylenimine (PEI) was for shBeclin1 delivery, and folate (FA) was employed as the tumor-targeting group. FPDP micelles demonstrated an average diameter of $161.9 \mathrm{~nm}$, good biocompatibility, applicable storage stability, excellent loading capacities for both DOX and shBeclin1 and a sustained drug release profile. In vitro cell culture experiments demonstrated that the uptake amount of FPDP/DOX micelles in folate receptor positive (FR ${ }^{+}$) HeLa cells was more than that in folate receptor negative (FR-) HepG2 cells, leading to significantly higher cytotoxicity against $\mathrm{FR}^{+} \mathrm{HeLa}$ cells. The simultaneous co-delivery of shBeclin1 and DOX to HeLa cells with FPDP micelles led to efficient reduction in the expression level of Beclin1 as well as synergistic cell apoptotic induction. Furthermore, in vivo studies revealed superior antitumor efficacy of tumor-targeted FPDP/DOX/shBeclin1 in comparison with non-FR-targeted PDP micelles and free DOX. These results highlighted that co-delivery of DOX and shRNA of Beclin1 with FPDP micelles has the potential to overcome the limitations of DOX in clinical cancer therapy.

\section{Introduction}

DOX is a leading, clinically used anticancer drug owing to its potency and broad spectrum of activity against diverse cancer types. ${ }^{1}$ However, its clinical therapeutic efficacy is greatly limited by its diverse toxicities to normal tissue, such as severe cardiotoxicity, ${ }^{2}$ and efflux pump-mediated or non-pump-related multi-drug resistance (MDR). During chemotherapy, the nonpump-related MDR which decreases the sensitivity of cancer cells to chemotherapeutic drugs has been predominantly attributed to the activation of anti-apoptotic cellular defense

${ }^{a}$ State Key Laboratory of Fine Chemicals, Dalian University of Technology, Dalian, 116024, P. R. China

${ }^{b}$ School of Life Science and Biotechnology, Dalian University of Technology, Dalian, 116024, P. R. China. E-mail: wangjingyun67@dlut.edu.cn; Fax:+86-41184706365; Tel: $+86-41184706356$

'Department of Environmental Health and Toxicology, School of Public Health, Dalian Medical University, Dalian, 116044, P. R. China.E-mail: liy76@yahoo.com; Fax: +8641186110329; Tel: +86-41186110330 pathways. $^{3}$ The autophagy, an evolutionarily conserved and selective degradation process of cellular components, can maintain energy production for cell survival under limited nutrient or toxic stimuli conditions. ${ }^{4,5}$ In many cancer cells, the autophagy process is reported to cause non-pump-related MDR, resulting in unsatisfactory chemotherapeutic effects with single drugs. $^{6,7}$ Thus, it is universally accepted that autophagydependent survival should be blocked to promote tumor-cell death. ${ }^{8}$ The autophagy process in tumor cells is a complicated biological process with a series of genes and mechanisms involved. Beclin1, as an autophagy-related gene, plays a vital role in autophagy activation by regulating the nucleation of autophagic vesicles. ${ }^{9,10}$ It has been revealed that Beclin1 is activated in the autophagy process and induces the occurrence of resistance mechanisms in chemotherapy. RNA interference (RNAi), a promising approach in cancer therapy, can selectively down-regulate relevant gene expression, overcome the multidrug resistance and improve the efficiency of chemotherapeutic drugs. ${ }^{11-14}$ RNAi is often mediated by short hairpin RNAs 
(shRNA) or small interfering RNAs (siRNA). The shRNA is a sequence of RNA which makes a tight hairpin turn and can be cloned into expression vectors to ensure that the shRNA is always expressed. Daniel et al. ${ }^{15}$ reported that partial Beclin1 silencing aggravates DOX and Fas induced apoptosis in HepG2 cells, demonstrating that selectively down-regulating Beclin1 gene expression can overcome the multi-drug resistance, and improve the sensitivity of cancer cells to DOX. However, in the research of Daniel's group, the silencing of Beclin1 gene expression and DOX treatment was administrated separately, and the therapeutic efficacy of DOX may be reduced. In this contribution, a nanocarrier-based delivery system offers a platform for the co-delivery of siRNA (shRNA) and anticancer chemotherapeutics simultaneously to the same tumor cells. ${ }^{16}$

In recent years, enormous efforts have been put into the development of novel siRNA (shRNA) and DOX co-delivery systems with enhanced bioavailability, low toxicity and prolonged circulation time, such as polymers, ${ }^{11,17}$ liposomes, ${ }^{18}$ dendrimers, ${ }^{19}$ mesoporous silica nanoparticles ${ }^{13,20,21}$ and quantum-dot (QD)-based nanoparticles. ${ }^{22}$ Among those systems, the amphiphilic cationic copolymers are widely used due to their excellent biocompatibility, desirable biodegradability, and unique self-assembling property. In general, the amphiphilic cationic copolymers can form core-shell structured micelles with a hydrophobic core and a hydrophilic shell of positive charge in aqueous solution, loading the hydrophobic antitumor drugs and polyanionic genes in single carrier. ${ }^{23,24}$ For instance, Chen et al. reported that FR-targeted multifunctional micelleplexes based on FA conjugated to poly(ethylene glycol)- block-poly(glutamic acid) (FA-PEG-PGA) loaded with Bcl-2 siRNA and DOX demonstrated a synergistic therapeutic effect in inhibiting the growth of $\mathrm{C} 6$ glioma in rats. ${ }^{25} \mathrm{~A}$ hyaluronic aciddecorated polyethylenimine-poly (D,L-lactide- $c o$-glycolide) (PEIPLGA) nanoparticle system was developed by Wang and coworkers for the co-delivery of DOX and miR-542-3p for triple negative breast cancer (TNBC) therapy and demonstrated improving antitumor efficacy. ${ }^{26}$

Pullulan, a natural non-ionic and linear homopolysaccharide, has attracted increasing attention in wide pharmaceutical and biomedical applications such as platform materials for drug and gene delivery systems due to its excellent biocompatibility, low viscosity and desirable water solubility. ${ }^{27-29}$ In our previous study, an amphiphilic polymer (named as PDP) was synthesized by linking lipophilic desoxycholic acid (DA) and low molecular weight (1 kDa) branched polyethylenimine (PEI) with pullulan (P). Herein, the amphiphilic polymer self-assembled into micelles with positively-charged PEI located on the hydrophilic shell to bind negatively charged genes through electrostatic interactions, and DA in the hydrophobic core to encapsulate hydrophobic anticancer drugs via hydrophobic interaction. PDP micelles could co-deliver tumor suppressor p53 gene and anticancer drug DOX, exhibiting effective antitumor efficacy in vitro and in vivo. ${ }^{30}$ Based on this encouraging results, in the present work, we modified PDP polymers with FA further to synthesize a new FR targeted pullulanbased copolymer (FPDP) (Scheme 1) and developed it as a FRtargeted micellar platform for cellular co-delivery of shBeclin1 and DOX to improve the anticancer effect of DOX through the blockade of autophagy process. The FR, a high affinity membrane
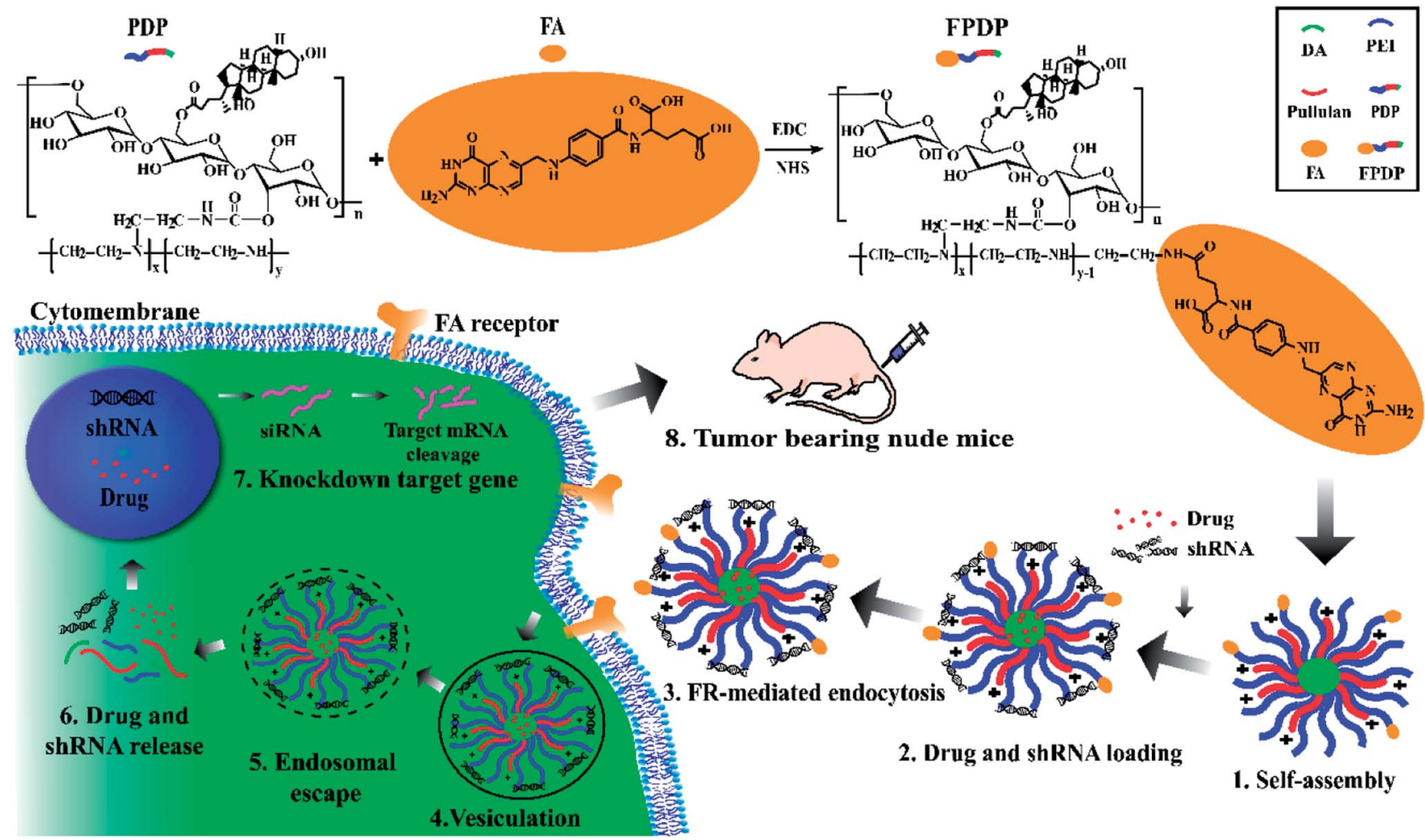

8. Tumor bearing nude mice

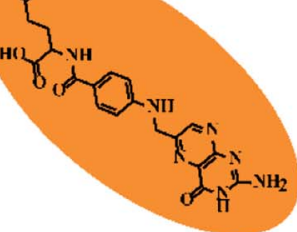

Scheme 1 Synthesis scheme of FPDP and illustration of tumor targeting strategy of FPDP nano-micelles for the simultaneous co-delivery of drug and shRNA into cancer cells. 
protein, is overexpressed in a wide variety of human tumors including more than $90 \%$ of ovarian carcinomas. ${ }^{31}$ Additionally, the expression level of $\mathrm{FR}$ in normal tissues is extremely low, ${ }^{32}$ making FR a useful marker for targeted drug/gene delivery to tumors. Therefore, nano-carriers modified with FA can be efficiently uptaken by the cancer cells overexpressing FR via receptormediated endocytosis due to the high affinity between FA and FR. ${ }^{33-36}$ To investigate the utility of FPDP micelle as co-delivery carrier for shBeclin1 and DOX in cancer therapy, the cytotoxicity and FR-targeted cellular uptake of FPDP/DOX micelles was evaluated against $\mathrm{FR}^{-}$HepG2 cells and $\mathrm{FR}^{+} \mathrm{HeLa}$ cells in vitro. Furthermore, shBeclin1 binding ability of FPDP micelles was investigated through gel retardation assay. More importantly, the antitumor efficacy of FPDP/DOX/shBeclin1 was assessed in HeLa cells and tumor-bearing nude mice to demonstrate that FPDP could be a promising candidate for prospective co-delivery of drugs and nucleic acids in cancer therapy.

\section{Materials and methods}

\subsection{Materials}

Folate (FA) was obtained from Sigma-Aldrich (St. Louis, USA). 1Ethyl-3-(3-dimethyllaminopropyl) carbodiimide hydrochloride (EDC $\cdot \mathrm{HCl}$ ), $N$-hydroxysuccinimide (NHS) and propidium iodide (PI) were obtained from Aladdin Industrial Corporation. Lipofectamine $^{\mathrm{TM}} 2000$ was obtained from Invitrogen. All other reagents were of analytical grade and used without further purification.

The shBeclin1 (sequence: GCTTCGCGCCGTAGTCTTA) and scrambled shRNA (shSCR) as a control (sequence: CTCAGGAGAGGAGCCATTT) were purchased from Genecopoeia Technologies Inc.

\subsection{Cell culture}

Human liver cell (HL7702), human cervical cancer cell (HeLa) and human hepatoma cell (HepG2) were purchased from the Cell Bank of Shanghai Institute of Biochemistry and Cell Biology. Cells were cultured in Dulbecco's modified Eagle's medium (DMEM) supplemented with 10\% (v/v) FBS under 5\% $\mathrm{CO}_{2}$ at $37{ }^{\circ} \mathrm{C}$ for $24 \mathrm{~h}$ until they reached $80 \%$ confluence.

\subsection{Synthesis of FPDP}

As shown in Scheme 1, the amphiphilic bifunctional pullulan derivative (PDP) was synthesized according to our previous published procedure. ${ }^{30}$ Then, $100 \mathrm{mg}$ FA was dissolved in $50 \mathrm{~mL}$ carbonate buffer solution (1 M, pH 9.6), $65.8 \mathrm{mg} \mathrm{EDC} \cdot \mathrm{HCl}$ and $39.5 \mathrm{mg}$ NHS was added. The mixture was stirred for $30 \mathrm{~min}$ at room temperature to activate the FA and PDP (366.6 mg) was added, the resultant mixture was stirred for further $24 \mathrm{~h}$ at $25{ }^{\circ} \mathrm{C}$. Afterwards, the reaction mixture was dialyzed against deionized water (MWCO, $10 \mathrm{kDa}$ ) for $72 \mathrm{~h}$ to wipe off small molecular weight impurities. Subsequently, the polymer FPDP solutions were lyophilized and stored at $4{ }^{\circ} \mathrm{C}$ until further use. FPDP structure and the degree of substitution of FA in the new polymer FPDP was determined by ${ }^{1} \mathrm{H}$ NMR (Varian INOVA400, USA).

\subsection{Preparation of micelles}

The blank FPDP micelles and FPDP/DOX micelles were prepared by dialysis method according to our previous published procedure. ${ }^{30}$ The preparation of FPDP/shBeclin1 complexes was achieved by electrostatic interaction. FPDP micelle solutions and shBeclin1 solutions in deionized water were mixed using gentle vortexing to form the FPDP/shRNA micelle complexes with different $\mathrm{N} / \mathrm{P}$ ratios (weight of FPDP/ weight of shRNA).

\subsection{Characterization and evaluation of micelles}

2.5.1. Characterization of FPDP and FPDP/DOX micelles. Critical micellar concentration (CMC) of FPDP was determined using pyrene as the probe by fluorescence spectroscopy (LS55, PerkinElmer). ${ }^{20,37-39}$

The particle size and zeta potential of FPDP, FPDP/DOX, FPDP/shBeclin1 and FPDP/DOX/shBeclin1 micelles were measured by dynamic light scattering (DLS) using Malvern Zetasizer NanoZS90 (Malvern instruments, UK). The morphology of micelles was observed by transmission electron microscopy (TEM, Tecnai G2 Spirit $120 \mathrm{kV}$ ). To evaluate the storage stability of the FPDP micelles, the particle size, PDI, and $\zeta$ potential were recorded for $60 \mathrm{~h}$.

2.5.2. Assessing the DOX loading ability of FPDP. The DOX loading capacity (LC) and encapsulation efficiency (EE) were calculated as: $\mathrm{LC} \%=$ (weight of DOX in micelles) $/$ (weight of polymeric micelles containing DOX) $\times 100 \%$ and $\mathrm{EE} \%=$ (weight of DOX in micelles)/(weight of DOX fed initially) $\times$ $100 \%$.

2.5.3. Evaluation of FPDP/shBeclin1 complexes. The formation of complexes between FPDP micelles and shBeclin1 was confirmed by electrophoresis retardation assay. Firstly, a series of FPDP/shBeclin1 micelle complexes with different N/P ratios were formed. Then, $10 \mu \mathrm{L}$ of FPDP/shBeclin 1 complexes ( $\operatorname{shBeclin} 1=1 \mu \mathrm{g}$ per lane) were electrophoresed on a $1 \%$ agarose gel containing $0.2 \mathrm{mg} \mathrm{mL}^{-1}$ ethidium bromide in trisacetate-ethylene (TAE) diaminetetraacetic acid buffer at $140 \mathrm{~V}$ for $40 \mathrm{~min}$. The gels were visualized and imaged under an ultraviolet transilluminator (G: BOX, Gene Company, Hong Kong, China).

\subsection{Hemolysis assay for biocompatibility evaluation}

The hemolytic ability of FPDP was evaluated by the method in our previous published procedure. ${ }^{30}$ All measurements were performed in triplicate and the percentage of hemolysis was calculated as: hemolysis $(\%)=\left(\mathrm{OD}_{\text {test }}-\mathrm{OD}_{\text {neg }}\right) /\left(\mathrm{OD}_{\text {pos }}-\mathrm{OD}_{\text {neg }}\right)$ $\times 100 \%$, where $\mathrm{OD}_{\text {test }}$, $\mathrm{OD}_{\text {neg }}$ and $\mathrm{OD}_{\text {pos }}$ are the absorbance values of the tested sample, positive control (water) and negative control (PBS) separately.

\subsection{In vitro drug release}

The in vitro release profiles of DOX from FPDP/DOX micelles were investigated by the dialysis method. ${ }^{38}$ FPDP/DOX containing $0.2 \mathrm{mg}$ of DOX was dissolved in $2 \mathrm{~mL}$ of PBS with different $\mathrm{pH}$ in a dialysis bag (MWCO, $10 \mathrm{kDa})$. Then, the 
dialysis bag was immersed in the corresponding pH PBS with shaking water bath at $37{ }^{\circ} \mathrm{C}$. At predetermined time intervals, withdraw $3 \mathrm{~mL}$ PBS and added the same volume of fresh PBS. The amount of DOX release were analyzed by fluorescence measurement with excitation at $485 \mathrm{~nm}$.

\subsection{In vitro cell culture studies}

2.8.1. The cytotoxicity evaluation by MTT. The cytotoxicity was studied by the MTT assays using HL7702, HeLa and HepG2 cells. Briefly, cells were seeded onto 96-well plate at $1.0 \times 10^{4}$ cells per well in DMEM for $24 \mathrm{~h}$. Then, the medium was replaced by fresh culture medium supplemented with $10 \%(\mathrm{v} / \mathrm{v})$ FBS containing different tested samples of the desired concentration. After the cells were incubated for another $24 \mathrm{~h}$ or $48 \mathrm{~h}$, MTT solution $\left(0.5 \mathrm{mg} \mathrm{mL}{ }^{-1}\right)$ was added and further incubated for $4 \mathrm{~h}$ at $37{ }^{\circ} \mathrm{C}$. Subsequently, the medium was removed, and then $100 \mu \mathrm{L}$ DMSO was added to dissolve the resulting purple crystals. The absorbance was measured at $570 \mathrm{~nm}$ and $630 \mathrm{~nm}$ using a microplate reader (Thermo Fisher Scientific). Cell viability was expressed as a percentage of the control culture value. Untreated cells in growth medium were used as the blank control.

2.8.2. The FR-targeted cellular uptake of FPDP/DOX. The FR-targeted cellular uptake behavior of free DOX and FPDP/ DOX micelles was investigated by confocal laser microscopy (CLSM) and flow cytometry (FCM) in $\mathrm{FR}^{+} \mathrm{HeLa}$ and $\mathrm{FR}^{-} \mathrm{HepG} 2$ cells. Briefly, HeLa or HepG2 cells were seeded onto 6-well plates at a density of $1.0 \times 10^{5}$ cells per well. After $24 \mathrm{~h}$, the cells were incubated with DOX and FPDP/DOX micelles at a final DOX concentration of $4.0 \mu \mathrm{g} \mathrm{mL} \mathrm{m}^{-1}$ for $8 \mathrm{~h}$. Then, for the visualization of free DOX and FPDP/DOX micelles in cells by CLSM, the cells were washed with PBS and stained with exclusive nuclear dye Hoechst 33342 at a final concentration of $10 \mu \mathrm{g}$ $\mathrm{mL}^{-1}$ for $30 \mathrm{~min}$. Fluorescence images of cells were obtained on an OLYMPUS FV 1000 (OLYMPUS, Japan). For quantifying by FCM, the cells were rinsed with PBS to remove micelles that were not ingested by the cells and then digested with pancreatin, centrifuged, re-suspended in $200 \mu \mathrm{L}$ PBS, and the fluorescence intensity of DOX was analyzed by flow cytometer (FACSCanto, BD, USA). The competitive inhibition of free FA was applied for verifying the FR-targeted uptake mechanism of FPDP/DOX. Cells were exposed to free FA of different concentrations for $30 \mathrm{~min}$ to occupy the FRs of the cell surface before HeLa or HepG2 cells were treated with DOX and FPDP/DOX (4.0 $\mu \mathrm{g} \mathrm{mL}^{-1}$ DOX-equivalent).

2.8.3. In vitro gene silencing experiment and western blot assay. For in vitro gene silencing experiment, HeLa cells were seeded onto 6 -well plate at a density of $1.0 \times 10^{5}$ cells per well in DMEM containing $10 \%$ (v/v) FBS for about $24 \mathrm{~h}\left(37^{\circ} \mathrm{C}, 5 \% \mathrm{CO}_{2}\right)$. Then the cells were washed three times with PBS, and incubated with FPDP/shSCR, free shBeclin1, lipo 2000/shBeclin1 and FPDP/shBeclin1 $\left(1.9 \mu \mathrm{g} \mathrm{mL} \mathrm{m}^{-1}\right.$ of shBeclin1 or shSCR at N/P 10) in fresh culture medium supplemented with $10 \%(\mathrm{v} / \mathrm{v})$ FBS for another $24 \mathrm{~h}$. Thereafter, the expression level of Beclin1 protein was evaluated by western blot assay. ${ }^{30}$
2.8.4. Apoptosis assay of FPDP/DOX/shBeclin1. After HeLa cells seeded onto 6-well plate were incubated with FPDP, free DOX, free shBeclin1, free shSCR, FPDP/DOX, FPDP/shBeclin1, FPDP/DOX + FPDP/shBeclin1, and FPDP/DOX/shBeclin1 (1.5

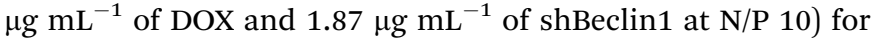
$24 \mathrm{~h}$ at $37^{\circ} \mathrm{C}$, cells were collected and re-suspended in $200 \mu \mathrm{L}$ of binding buffer. Finally, annexin V-FITC $(5 \mu \mathrm{L})$ and PI $(5 \mu \mathrm{L})$ were added and mixed for $15 \mathrm{~min}$ in the dark. The stained cells were analyzed using a flow cytometer with the untreated cells as control.

\subsection{In vivo studies}

2.9.1. Antitumor efficacy and tumor target of FPDP/DOX. Female BALB/C nude mice (6-8 week-old) were inculcated with HeLa cells to establish subcutaneous human cervical cancer xenografts models for in vivo antitumor efficacy experiments. Briefly, tumors were implanted by injecting $5 \times 10^{6}$ HeLa cells suspended in a $100 \mathrm{~mL}$ PBS solution subcutaneously into the mice, respectively. When the tumor reached a volume of approximately $0.5 \mathrm{~cm}$ in diameter, the mice were randomly divided into 11 groups $(n=6)$, which separately received different treatment (DOX concentration of $4 \mathrm{mg} \mathrm{kg}^{-1}, 4.98 \mathrm{mg}$ $\mathrm{kg}^{-1}$ of shBeclin1 at $\mathrm{N} / \mathrm{P}=10$ ) by intravenously injecting via tail vein each two days. The tumor sizes and mice weights were measured after injection every time, and three dimensions of solid tumors were measured by using a caliper, followed by a formula: tumor volume $\left(\mathrm{cm}^{3}\right)=(1 / 2) a b^{2}$ ( $a=$ tumor length, $b=$ tumor width). Additionally, after $12 \mathrm{~h}$ post-injection, the uptake and distribution of free DOX and FPDP/DOX in vivo was also visualized, and the images of living mice were obtained by small animal in vivo imaging system (NightOWL II LB983, Berthold Technologies, Germany) equipped with a sensitive Charge Coupled Device (CCD) camera. All animal procedures were performed in accordance with the Guidelines for Care and Use of Laboratory Animals of Dalian University of Technology and experiments were approved by the Animal Ethics Committee of Dalian University of Technology.

2.9.2. Hematoxylin and eosin (H\&E) assay. For histological analysis, the HeLa cell bearing nude mice were sacrificed on 21 days after injection of tested samples, the major organs and tumors were collected and fixed in $4 \%$ paraformaldehyde overnight. The paraffin embedded specimens were cut at $5 \mu \mathrm{m}$ thick sections, de-waxed and rehydrated with xylene, a graded series of ethanol, and deionized water successively. ${ }^{40}$ Finally, the fixed sections were stained with H\&E to assess histological alterations by OLYMPUS FV 1000 microscope (OLYMPUS, Japan).

2.9.3. Immunohistochemical analysis. For immunohistochemical analysis, tumor sections of paraffin-embedded were prepared and processed as described previously. ${ }^{30,41}$ Beclin1 protein expression in the sections was detected with an antiBeclin1 monoclonal antibody (Beclin1, 1 : 100 dilution, Proteintech, US). A Beclin1-positive reaction was indicated by a reddish-brown precipitate in the cytoplasm and analyzed by OLYMPUS FV 1000 microscope. 


\subsection{Statistical analysis}

All the experiments were performed three times and the data were presented as means \pm standard deviation (SD). Statistical analysis was conducted by using the Student's $t$-test and oneway analysis of variance analysis (ANOVA) by Origin version 9.0 software (Northampton, USA). The difference was considered to be statistically significant when $P$ value $<0.05(*)$ and $P$ value $<0.01(* *)$.

\section{Results and discussion}

\subsection{Synthesis and characterization of FPDP and FPDP/DOX micelles}

FPDP polymer was synthesized according to the route in Scheme 1. As shown in Fig. 1A, in comparison with the ${ }^{1} \mathrm{H}$ NMR of PDP, the new chemical shifts at 6.5-8.0 ppm of FPDP were associated with aromatic protons of FA, indicating that FPDP polymer was successfully synthesized. In this paper, the substitution degree of FA means the number of FA substituents for per 100 glucose unit, and the calculated substitution degree of FA in polymer FPDP was 2.2.

Next, we investigated the self-assembly behavior and characterized FPDP micelles. CMC is an essential parameter related to the self-assembly ability of amphiphilic polymers. In this study, the CMC of FPDP micelles was determined to be $12.15 \mathrm{mg} \mathrm{L}^{-1}$. The relatively low CMC value suggested that the micelles would retain structurally intact under extensively diluted conditions after administration, which was advantageous to prolong blood circulation time and avoid the advanced leakage of drugs. Additionally, as shown in Fig. 1C, the particle sizes and zeta potentials of FPDP micelles didn't display obvious change after FPDP micelles were incubated in 10\% serum for different storage time, demonstrating that FPDP micelles have good storage stability.

DLS measurement demonstrated that the mean size of blank FPDP micelles was $161.9 \pm 1.45 \mathrm{~nm}$, and the morphology of FPDP micelles observed by TEM (Fig. 1D) showed that blank FPDP micelles were uniformly dispersed and regularly spherical. Importantly, the positive surface zeta potential $(30.33 \pm 1.0$ $\mathrm{mV}$ ) of FPDP micelles would provide negatively-charged genebinding ability to the carrier.

The biocompatibility of FPDP was first assessed by the hemolysis test. As demonstrated in Fig. 1E, the hemolysis percentage caused by FPDP was still less than $5 \%$ even at higher FPDP concentration of $2.0 \mathrm{mg} \mathrm{mL}{ }^{-1}$. It was reported that hemolysis rate less than $5 \%$ is acceptable for biomedical
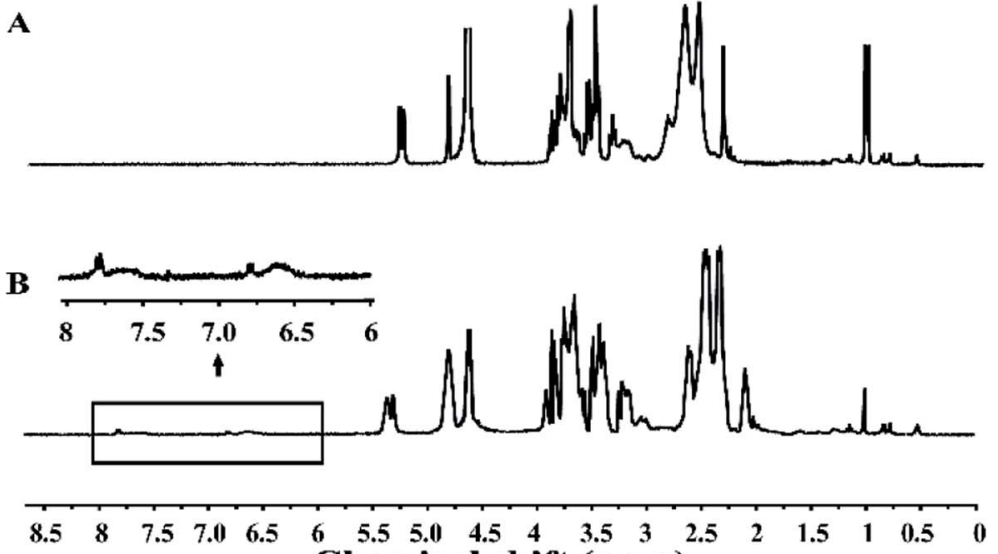
Chemical shift (ppm)

D

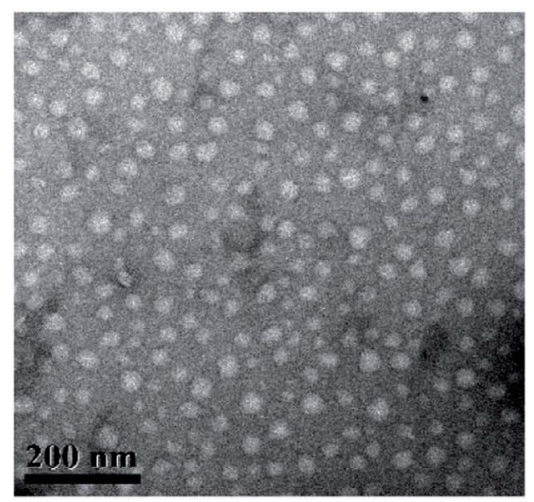

$\mathbf{E}$

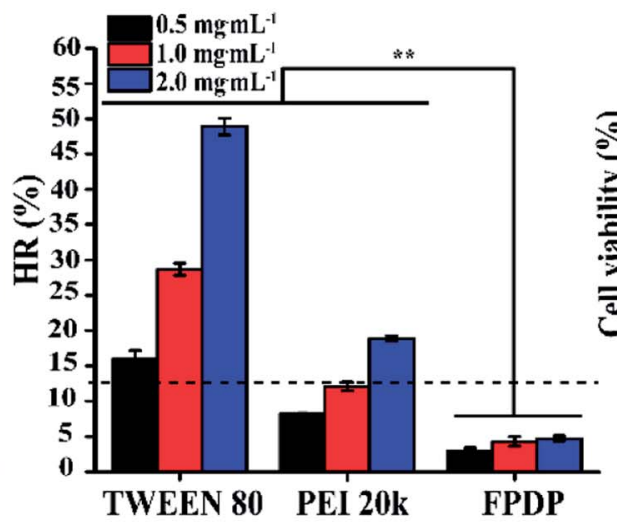

$\mathbf{F}$
C
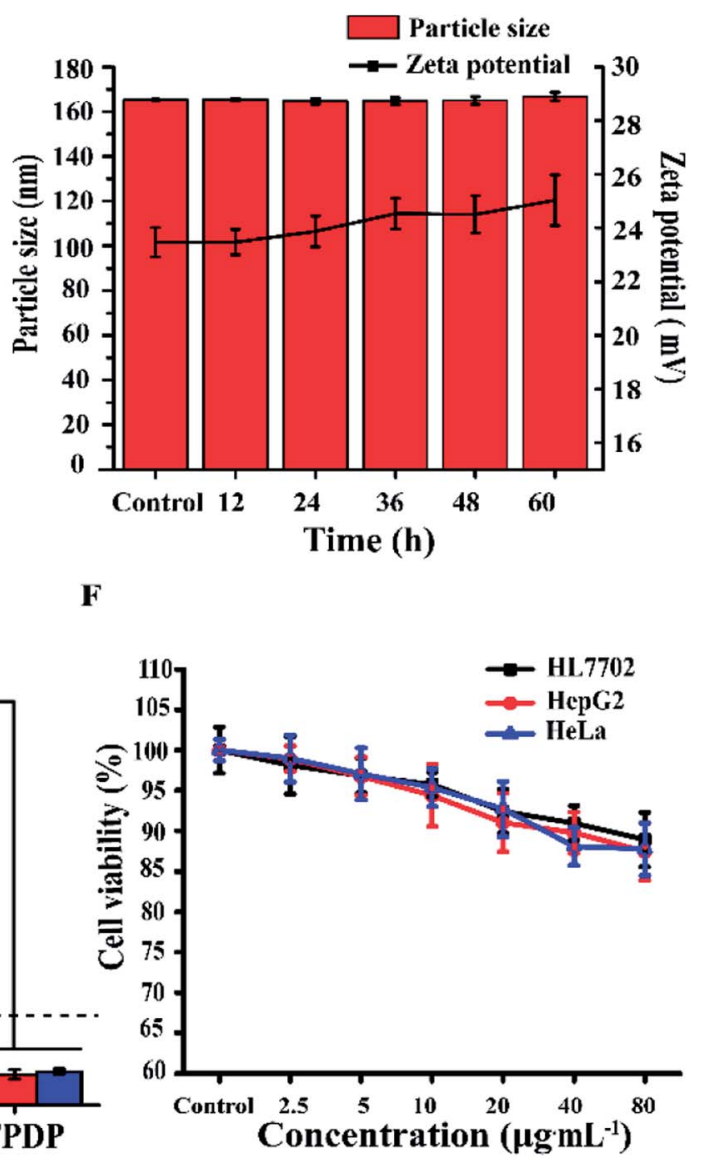

Fig. 1 Characterization of FPDP micelles. ${ }^{1} \mathrm{H}$ NMR spectra of (A) PDP and (B) FPDP; (C) stability of FPDP micelles in 10\% serum; (D) transmission electron microscopy images of FPDP (E) hemolysis test results different concentrations of FPDP after incubation with red blood cells suspension of sheep blood for $3 \mathrm{~h}$; (F) viability of different cells assayed by MTT after the cells were treated with FPDP at different concentrations for $48 \mathrm{~h}$. 
application, ${ }^{39}$ thus FPDP polymer displayed good blood compatibility and is suitable for drug delivery.

Furthermore, the biocompatibility of FPDP was evaluated by its cytotoxicity against non-cancer cells (HL7702 cells) and two cancer cells (HeLa and HepG2 cells). As demonstrated in Fig. 1F, even at a high FPDP concentration $\left(80 \mu \mathrm{g} \mathrm{mL} \mathrm{m}^{-1}\right)$, the cell viability for all three tested cell lines still maintained higher than $85 \%$, manifesting that blank FPDP micelles were non-toxic to HL7702, HeLa and HepG2 cells. In one word, the low cytotoxicity and hemolysis rate of FPDP copolymer makes it potential candidate as a safe material for gene/drug delivery.

\subsection{DOX release behavior, FR-targeted cellular uptake and cytotoxicity of FPDP/DOX micelles in vitro}

In this study, the FPDP/DOX micelles were prepared by dialysis method, and the drug loading capacity and encapsulation efficiency of the FPDP micelles for DOX were determined to be $7.4 \pm$ $0.02 \%$ and $81.4 \pm 0.17 \%$ separately. The in vitro release behavior of DOX was evaluated in PBS solutions with $\mathrm{pH}$ values of 7.4, 6.5, 5.0 respectively, and free DOX was used as the control. As shown in Fig. 2A, free DOX exhibited rapid release rate, and was completely released within $6 \mathrm{~h}$. However, the FPDP/DOX micelles showed sustained release of DOX over $96 \mathrm{~h}$, this phenomenon was due to the shielding effects of FPDP to DOX. On the other hand, the release rate of DOX from FPDP/DOX micelles increased remarkably in a pH-dependent manner. For example, at $48 \mathrm{~h}$ period, DOX release amount was only $54.47 \%$ at $\mathrm{pH} 7.4$, but reached up to $60.59 \%$ and $71.07 \%$ at $\mathrm{pH} 6.0$ and $\mathrm{pH} 5.0$ respectively. One reason is that the protonation of the amino groups of PEI in FPDP under slightly acid environment changed the hydrophobic and hydrophilic properties of FPDP, which made the micelles to change from a compact sphere to an expanding shape, leading to the release of DOX more easily. Additionally, DOX was also protonated at acidic $\mathrm{pH}$ values, owing to its $\mathrm{p} K_{\mathrm{a}}$ of $8.3,{ }^{42}$ and the increasing solubility of protonated DOX in the micelles also induces easier drug release. It was reported that cancer cells intracellular environment is characterized by lower $\mathrm{pH}(4.5-7.2)$ as compared with normal cells. ${ }^{43,44}$ Thus, the $\mathrm{pH}-$ sensitive release behavior is believed to prevent DOX release during the blood circulation ${ }^{46}$ and is favorable for inducing sufficient release of DOX in the acidic endosome/lysosome compartment after internalization by the cancer cells.

In order to confirm that FPDP/DOX micelles can target $\mathrm{FR}^{+}$ cells, the cell uptake behavior of FPDP/DOX in $\mathrm{FR}^{+} \mathrm{HeLa}$ cells and $\mathrm{FR}^{-}$HepG2 cells after incubation with free DOX or FPDP/DOX micelles $\left(4.0 \mu \mathrm{g} \mathrm{mL} \mathrm{mL}^{-1}\right.$ DOX-equivalent) for $8 \mathrm{~h}$ was visualized under CLSM and quantitatively measured by FCM respectively. Fig. 2B and $\mathrm{C}$ demonstrated that in comparison with free DOX groups, both $\mathrm{FR}^{+}$HeLa and $\mathrm{FR}^{-}$HepG2 cells with FPDP/DOX groups showed higher DOX fluorescent signals, demonstrating that FPDP/DOX had higher cellular uptake efficiency than free DOX. Moreover, the fluorescence image of FPDP/DOX-incubated $\mathrm{FR}^{+}$HeLa cells was much brighter compared to that of FPDP/ DOX-incubated $\mathrm{FR}^{-}$HepG2 cells, displaying that much more FPDP/DOX entered $\mathrm{FR}^{+}$HeLa cells and reflecting an enhanced targeted binding ability of FPDP/DOX to $\mathrm{FR}^{+}$HeLa cells.
Meanwhile, in order to check whether the enhanced uptake of FPDP/DOX against $\mathrm{FR}^{+}$HeLa cells was FR mediated endocytosis or not, both HeLa and HepG2 cells were treated with different concentration of free $\mathrm{FA}\left(0,1,2,5 \mathrm{mg} \mathrm{mL}^{-1}\right)$ for $30 \mathrm{~min}$ before consequent incubation with FPDP/DOX $\left(4.0 \mu \mathrm{g} \mathrm{mL} \mathrm{m}^{-1}\right.$ DOXequivalent) for $8 \mathrm{~h}$. As displayed in Fig. 2E, almost no DOX fluorescence was observed after the HeLa cells were pretreated with $5 \mathrm{mg} \mathrm{mL}^{-1} \mathrm{FA}$. By contrast, the presence of FA did not show obvious effect on the uptake of FPDP/DOX in $\mathrm{FR}^{-} \mathrm{HepG} 2$ cells. As seen from Fig. 2F, the fluorescent signals of DOX in $\mathrm{FR}^{+} \mathrm{HeLa}$ cells depended significantly on the amount of FA in the culture. The fluorescent intensity in $\mathrm{FR}^{+} \mathrm{HeLa}$ cells without the pretreatment of FA was the strongest, and with increasing the amount of FA, the red fluorescent density of DOX in HeLa cells decreased gradually. All these results revealed that excess free FA molecules blocked the cellular uptake of the FA-decorated FPDP/ DOX nanomicelles by competitive binding to the folate receptors on $\mathrm{FR}^{+} \mathrm{HeLa}$ cell surface, demonstrating that FPDP/DOX nanomicelles can effectively target FR-positive cancer cells via the FRmediated endocytosis process.

Finally, the cytotoxicity of FPDP/DOX in $\mathrm{FR}^{+} \mathrm{HeLa}$ cells and $\mathrm{FR}^{-}$HepG2 cells were analyzed using MTT method. As demonstrated in Fig. 2D, in $\mathrm{FR}^{+}$HeLa cells, FPDP/DOX micelles displayed higher cytotoxicity in comparison with free DOX, and $\mathrm{IC}_{50}$ of FPDP/DOX calculated as $1.28 \mu \mathrm{g} \mathrm{mL} \mathrm{mL}^{-1}$ was lower than that of free DOX $\left(3.73 \mu \mathrm{g} \mathrm{mL} \mathrm{m}^{-1}\right)$. But there was no obvious cytotoxicity difference between free DOX and FPDP/DOX micelles treated $\mathrm{FR}^{-}$HepG2 cells. More excitedly, the FPDP/ DOX micelles showed higher cytotoxicity in $\mathrm{FR}^{+} \mathrm{HeLa}$ cells than that in $\mathrm{FR}^{-} \mathrm{HepG} 2$ cells, and $\mathrm{IC}_{50}$ of FPDP/DOX against HeLa cells $\left(1.28 \mu \mathrm{g} \mathrm{mL}^{-1}\right)$ was lower than that in HepG2 cells $\left(3.51 \mu \mathrm{g} \mathrm{mL}{ }^{-1}\right)$. These results were in coincidence with the high cellular uptake efficiency of FPDP/DOX by $\mathrm{FR}^{+} \mathrm{HeLa}$ cells and highlighted the FR-mediated targeted endocytosis process of FPDP/DOX in the enhanced cytotoxic activity.

\subsection{ShRNA-binding and gene silencing in vitro}

The positive surface zeta potential of micelles is important for gene-binding and internalizing into cancer cells. In the present study, the zeta potentials of FPDP/DOX, FPDP/shBeclin1 and FPDP/DOX/shBeclin1 micelles were $29.75 \pm 0.4 \mathrm{mV}, 28.28 \pm$ $1.1 \mathrm{mV}$ and $26.09 \pm 1.1 \mathrm{mV}$ respectively.

To examine the ability of FPDP and FPDP/DOX micelles to bind shBeclin1 plasmid, agarose gel electrophoresis was performed to check the retardation of shBeclin1 plasmid mobility by FPDP and FPDP/DOX micelles. From Fig. 3A and Fig. 3B, it was found that both FPDP and FPDP/DOX could retard completely the electrophoretic mobility of shBeclin1 plasmid when the N/P reached to 10 , pointing to an obvious binding ability to shBeclin1 plasmid. These results indicated that both FPDP and FPDP/DOX could form stable and compact complexes with shBeclin1 plasmid when mass ratio was equal to or higher than 10 . Therefore, $\mathrm{N} / \mathrm{P}$ ratio of 10 was used in the subsequent drug/gene co-delivery experiments.

Genetic studies have identified that in cancer cells exposed to antitumor agents, Beclin1 protein plays a crucial role in the 
autophagy activation by regulating the nucleation of autophagic vesicles to support cancer cell survival. ${ }^{10}$ Therefore, concomitantly using shRNA to block the Beclin1 protein-relevant signaling pathway offers an effective solution to overcome the antiapoptotic defense of cancer cells caused by drug-induced autophagy. ${ }^{45}$ In this study, the expression level of Beclin1 protein blocked by shBeclin1 was used to assess FPDP-mediated shBeclin1 delivery in HeLa cells by western blot experiment. As indicated in Fig. 3C and D, non-
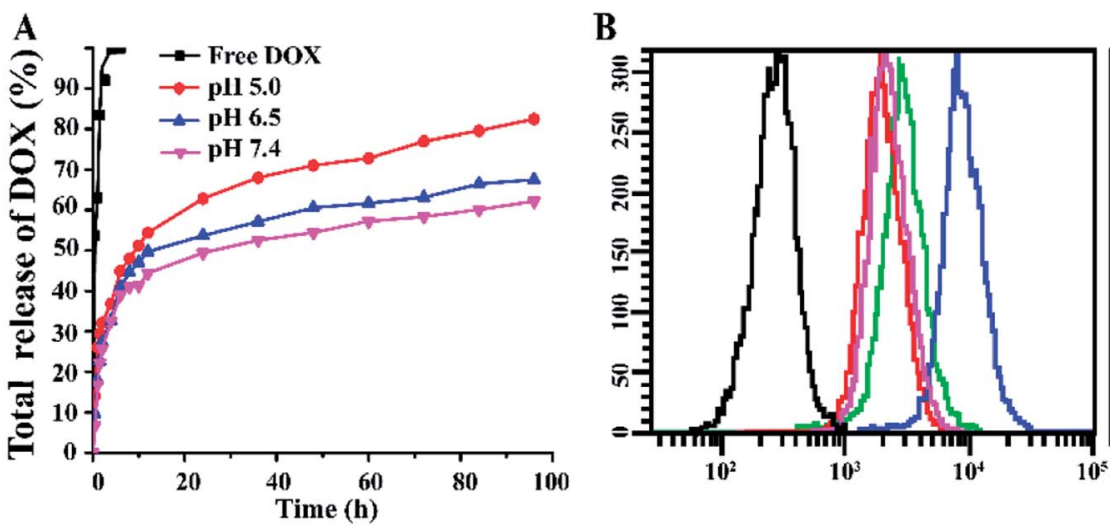

\begin{tabular}{|c|c|}
\hline Group & Fluorescence intensity \\
\hline - Control & $282 \pm 20.1$ \\
\hline - HepG2-Free DoX & $3633 \pm 128.2$ \\
\hline -HepG2-FPDP/DOX & $4526.7 \pm 322.9$ \\
\hline - HeLa-Free DOX & $3239 \pm 581.1$ \\
\hline - HeLa-FPDP/DOX & $8337.3 \pm 656.3$ \\
\hline
\end{tabular}

C

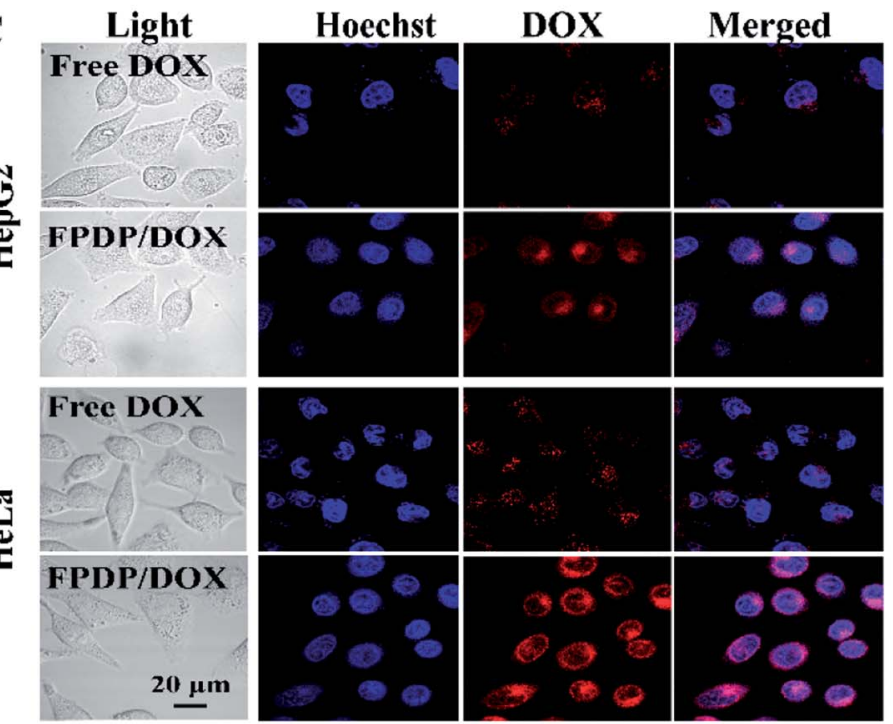

$\mathbf{E}$
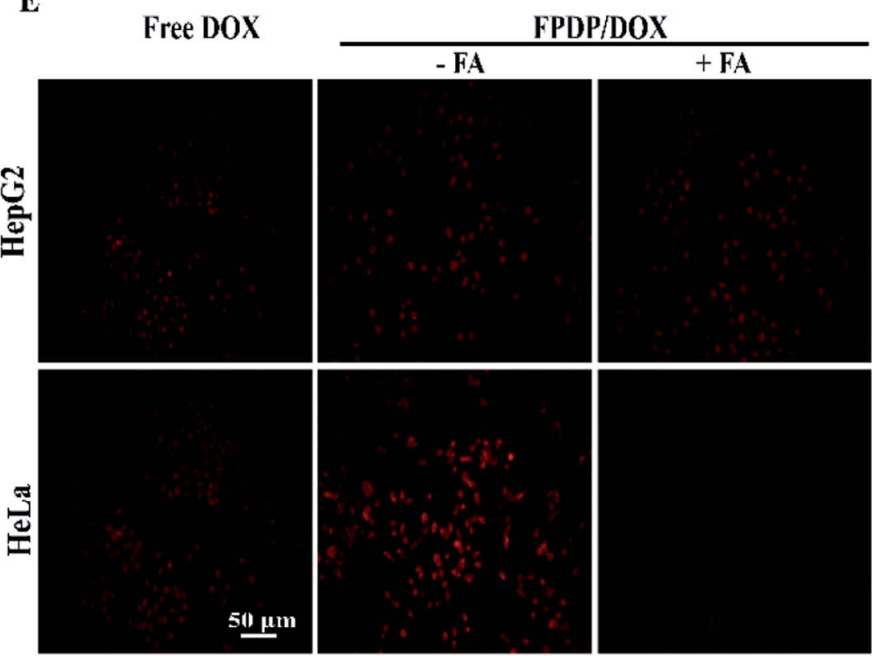

D
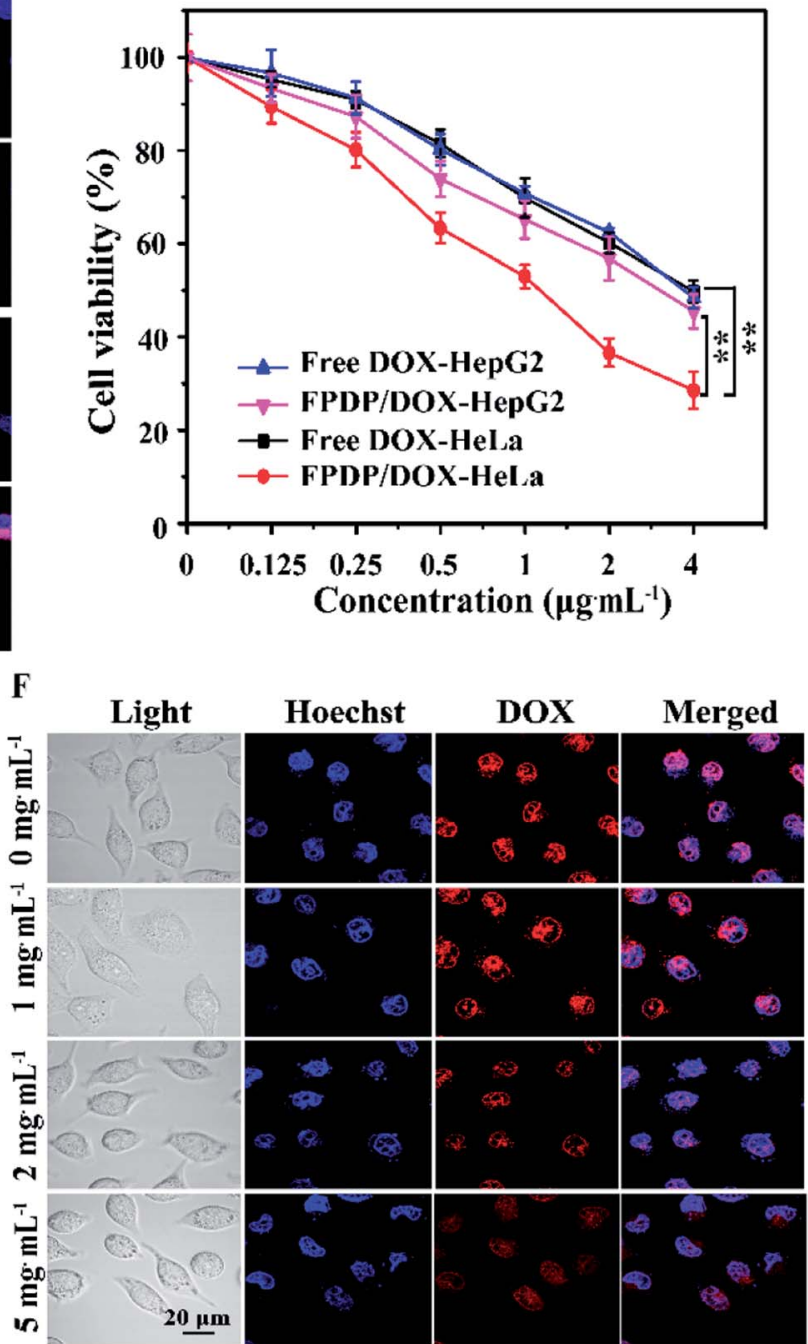

Fig. 2 (A) DOX release profiles of FPDP/DOX micelles at different $\mathrm{pH}$; (B) the cellular uptake of free DOX and FPDP/DOX micelles by HeLa and HepG2 cells assayed by a flow cytometer and (C) by CLSM imaging (blue represents nuclei and red represents DOX); (D) cytotoxicity of free DOX and FPDP/DOX micelles at different DOX concentrations after incubation of $24 \mathrm{~h}$; (E) the cellular uptake of free DOX and FPDP/DOX micelles for $8 \mathrm{~h}$ against HeLa and HepG2 cells after treatment with or without folic acid $\left(5 \mathrm{mg} \mathrm{mL}^{-1}\right)$; (F) the cellular uptake of FPDP/DOX by HeLa cells preincubated in culture medium containing different concentrations of FA. 
targeted shRNA (shSCR) showed no gene silencing effect on Beclin1 protein expression, but the targeted FPDP/shBeclin1 complexes almost completely knocked down the expression of Beclin1 protein. Free shBeclin1 also exerted no obvious influence on the expression of Beclin1 protein, which was mainly due to the poor cellular uptake efficiency of free shBeclin $1 .{ }^{46}$ In a word, FPDP has the potential to be an idealistic shRNA delivery carrier for future gene silencing in the treatment of cancer.

\subsection{Co-delivery of DOX and shBeclin1 in vitro}

The experimental results above demonstrated that FPDP polymer holds the potential application for the targeted delivery of DOX and shBeclin1 into the $\mathrm{FR}^{+}$cancer cells separately. Herein, we further investigated the synergistic antitumor effect of the co-delivery of DOX and shBeclin1 against HeLa cells. Firstly, the apoptosis of HeLa cells induced by FPDP/DOX/shBeclin1 was analyzed by Annexin V-FITC and PI double staining via FCM. As illustrated in Fig. 4A, there were only $20.3 \%$ apoptotic and dead cells induced by free DOX. By contrast, FPDP/DOX enhanced the accessibility of DOX to HeLa cells and induced higher apoptosis rate $(39.4 \%)$. On the other hand, the rates of apoptotic cells in free shBeclin1 (3.6\%) and FPDP/shBeclin1 (4.9\%) groups didn't show obvious difference in comparison with control group, demonstrating that the single delivery of shBeclin1 failed to afford therapy efficacy. However, co-delivery of DOX and shBeclin1 led to total $85.9 \%$ apoptotic rate of HeLa cells, which was remarkably higher than any other groups. By contrary, FPDP/ DOX + FPDP/shBeclin1 group only induced $46.4 \%$ apoptotic and dead HeLa cells owing to the fact that FPDP/DOX and FPDP/shBeclin1 could not enter the same cancer cells simultaneously. Taken together, these finding suggested that the codelivery of shBeclin1 and DOX with FPDP micelles enabled the silence of Beclin1 and suppressed the autophagy caused by DOX chemotherapy, consequently make cancer cells more sensitive to DOX, and thus induce cell-apoptosis more efficiently.

Additionally, the synergetic antitumor effect of DOX and shBeclin1 was further investigated by evaluating the cytotoxicity with MTT method. As displayed in Fig. 4B, after incubation for $24 \mathrm{~h}$, blank FPDP, free shSCR and FPDP/shSCR treated HeLa cells showed no obvious toxicity compared with control group. Whereas, FPDP/DOX/shBeclin1 emerged significantly higher cytotoxicity compared with other treatment, demonstrating that FPDP micelles could achieve efficiently co-delivery of shBeclin1 and DOX into the same cells to induce higher cell apoptosis rate and improve the anticancer effect of DOX through the blockade of autophagy process.

\subsection{Antitumor efficacy of FPDP/DOX/ShBeclin1 in vivo and systemic toxicity evaluation of FPDP/DOX/shBeclin1}

The positive antitumor effect of FPDP/DOX/shBeclin1 in vitro encouraged us to further investigate the synergistic antitumor therapeutic efficacy of FPDP/DOX/shBeclin1 in HeLa tumorbearing $\mathrm{BALB} / \mathrm{c}$ nude mice. It could be observed in Fig. 5A that FPDP/DOX/shBeclin1 micelles displayed the highest efficiency in inhibiting the tumor growth. Fig. 5B showed that the tumors in PBS and FPDP/shBeclin1 group grew quickly, the
A

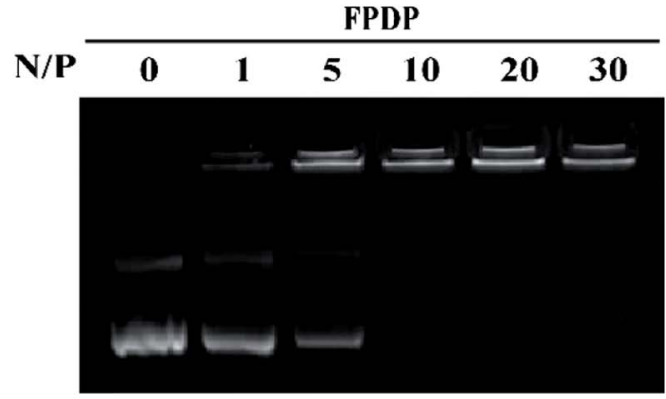

C

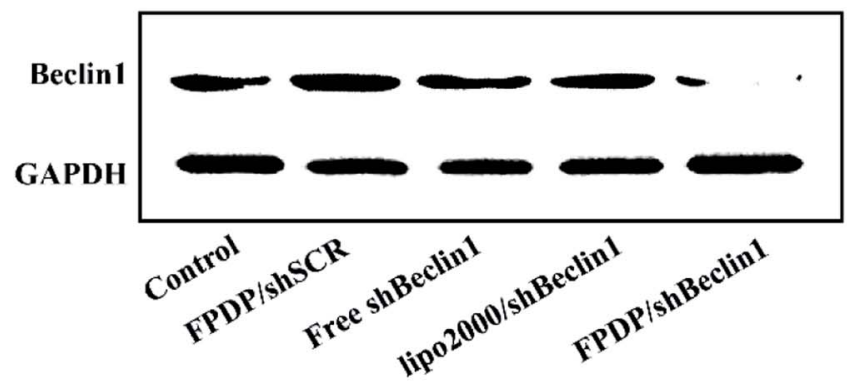

B

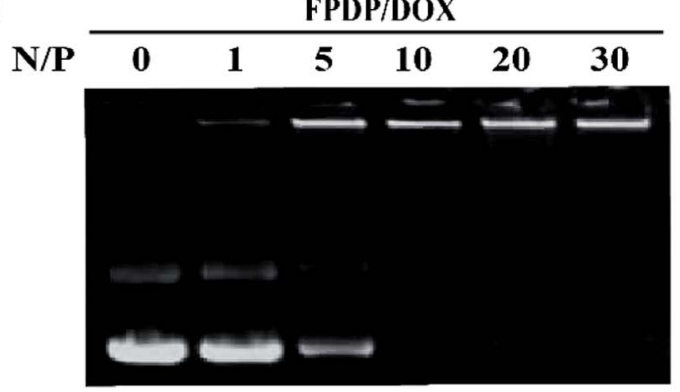

D

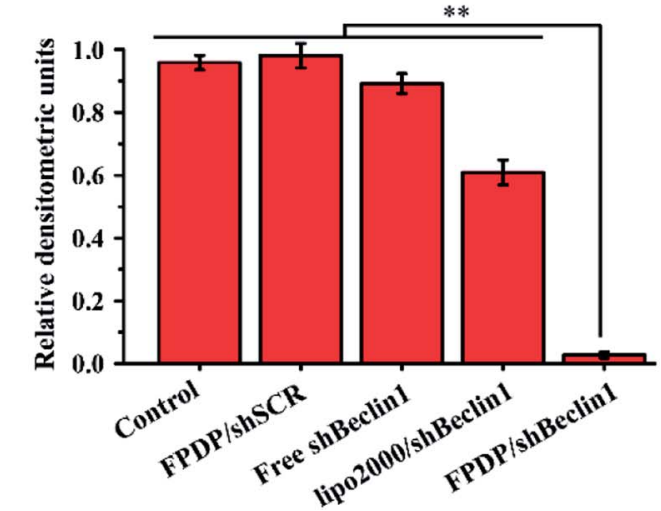

Fig. 3 ShRNA-binding of FPDP or FPDP/DOX micelles and transfection ability of FPDP/shRNA micelles in vitro. (A) Agarose gel electrophoresis retardation assay of shRNA by FPDP and (B) FPDP/DOX; (C) western blotting analysis for Beclin1 protein expression of HeLa cells after different treatment. (D) The intensity of Beclin1 expression quantified using Gel-Pro analyzer software. In experiments, shSCR and shBeclin1 concentration was at $1.87 \mu \mathrm{g} \mathrm{mL}^{-1}, \mathrm{~N} / \mathrm{P}=10$. 
A

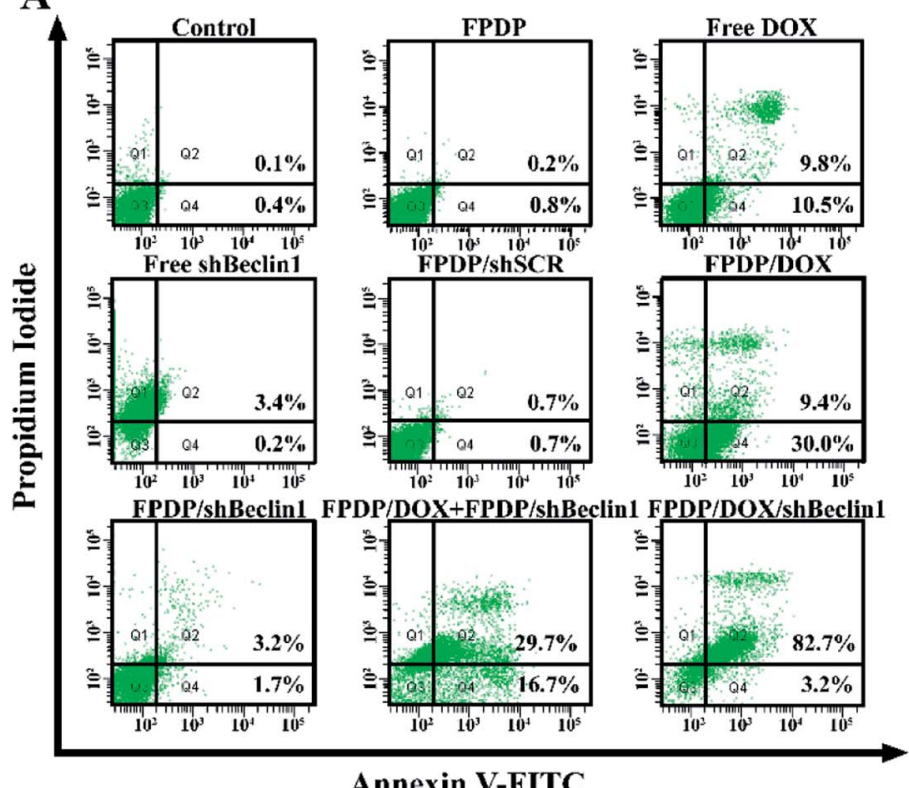

B

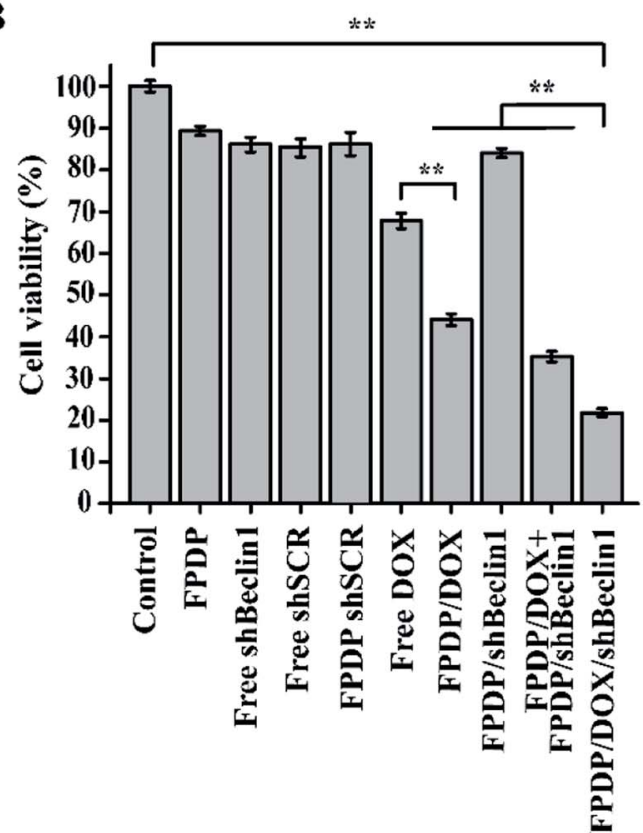

Fig. 4 In vitro antitumor effect of DOX and shBeclin1 co-delivery on HeLa cells incubated with different treatment by apoptosis analysis and MTT. (A) Apoptosis analysis by FCM; (B) cell viability assayed by MTT. In experiments, DOX concentration was set at $1.5 \mu \mathrm{g} \mathrm{mL}^{-1}$; shSCR and shBeclin1 concentration was at $1.87 \mu \mathrm{g} \mathrm{mL}^{-1}, \mathrm{~N} / \mathrm{P}=10$.

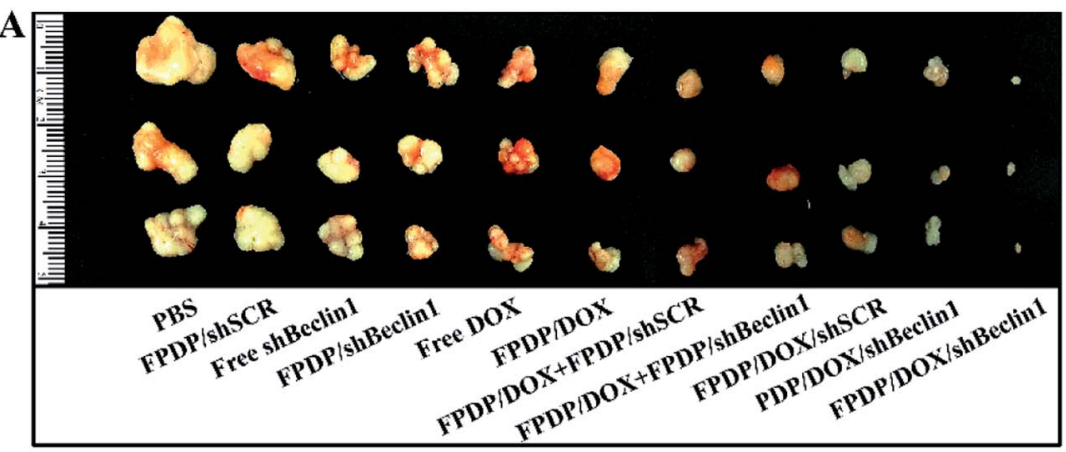

C

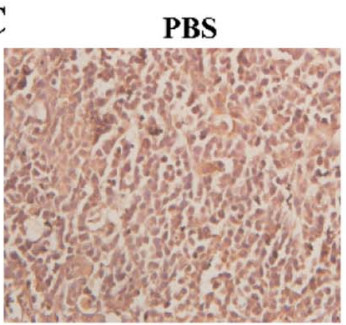

FPDP/DOX+FPDP/shBeclin 1

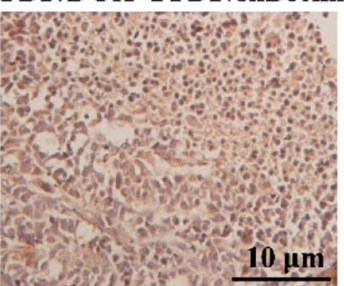

FPDP/shBeclin1

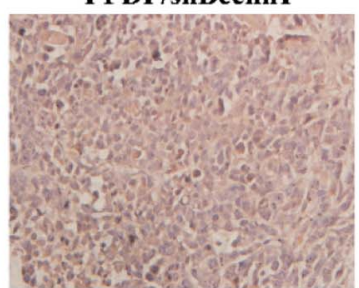

PDP/DOX/shBeclin1

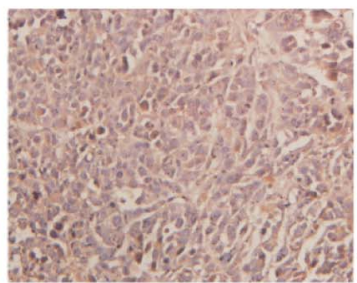

FPDP/DOX

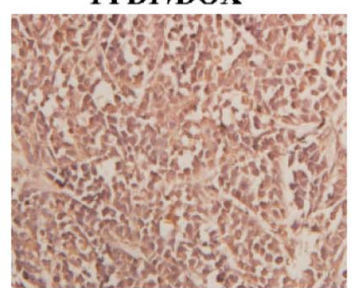

FPDP/DOX/shBeclin1

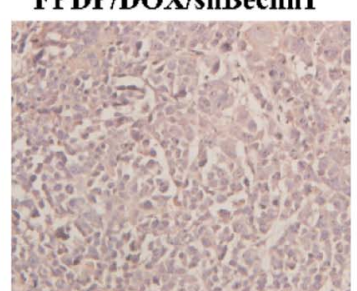

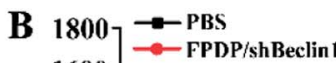

$1600-\longrightarrow$ FPDP/DOX

$1400-\checkmark-$ FPDP/shSCR+FPDP/DOX

当 $1200-\multimap$ FPDP/DOX/shSCR

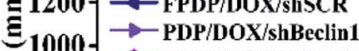

$1000-$ FPDP $/ D O X / \mathrm{shBcclin} 1$

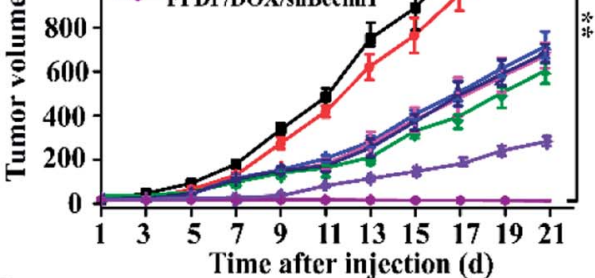

D

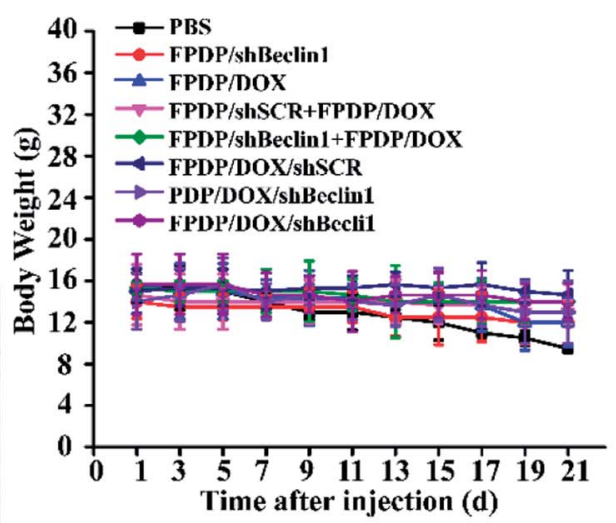

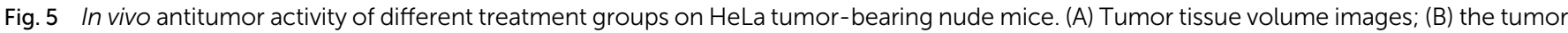
growth curve; (C) immunohistochemistry of Beclin1 performed in tumor sections; (D) body weight change of mice. 
average tumor volume reached approximately $1300 \mathrm{~mm}^{3}$ and $1200 \mathrm{~mm}^{3}$ within 21 days respectively, indicating that the knockdown of Beclin1 gene alone exerted no effect on inhibiting the growth of tumors. The mice treated with other micelles (FPDP/DOX, FPDP/DOX + FPDP/shSCR, FPDP/DOX + FPDP/ shBeclin1, and PDP/DOX/shBeclin1) showed gradual tumor growth at various rates. As we expected, the tumors in FRtargeted FPDP/DOX/shBeclin1 group exhibited almost no growth during the whole therapeutic process.

Moreover, to investigate the role of Beclin1 knockdown in synergistic antitumor efficacy, we sectioned tumor xenografts and analyzed the expression level of shBeclin1 protein by immunohistochemistry. In Fig. 5C, a Beclin1-positive reaction was indicated by a reddish-brown precipitate in the cytoplasm and deeper color means more Beclin1 protein was expressed. FPDP/DOX group showed obvious deeper reddish-brown color in the cytoplasm compared with PBS group, due to the DOXinduced autophagy activation, which result in the upregulation of Beclin1 protein expression. In contrast, owing to the knockdown effect of shBeclin1, the expression of Beclin1 protein in FPDP/DOX/shBeclin1 group was markedly downregulated, and also was lower than that in FPDP/shBeclin1 and PDP/DOX/shBeclin1 micelles group, demonstrating the excellent tumor targeting ability of FPDP micelles.
As is well-known, most chemotherapeutic agents can cause severe side effects such as organ toxicity and weight loss, thus compromising the therapeutic efficacy. From Fig. 5D, we can see that within 21 days the body weight of mice treated with FPDP/ DOX/shBeclin1 showed gradually increased, suggesting low toxicity of injected nano-complexes. Fig. 6 showed HE staining on heart, lung, liver, spleen and kidney, in comparison with the organs in control PBS group, no significant histopathological damages were observed in the tissues of the organ treated with micelles-treated groups. Therefore, these results demonstrated that the co-delivery of DOX/shBeclin1 by FPDP micelles can not only enhance the anti-tumor efficacy of DOX through overcoming the drug-resistance caused by DOX-induced autophagy, but also reduced its systemic toxicity, indicating that FPDP/DOX/ shBeclin1 will be a promising therapeutic method for cancer therapy in the future.

\subsection{Targeting ability of FPDP in vivo}

The targeting ability of FPDP nanocomplexes in tumor-bearing mice in vivo was also checked, and displayed in Fig. 7. In free DOX-treated group, the DOX intensity in tumor was weak and appeared at several sites, meaning that free DOX can distribute in the whole body of mice and be metabolized quickly. However,

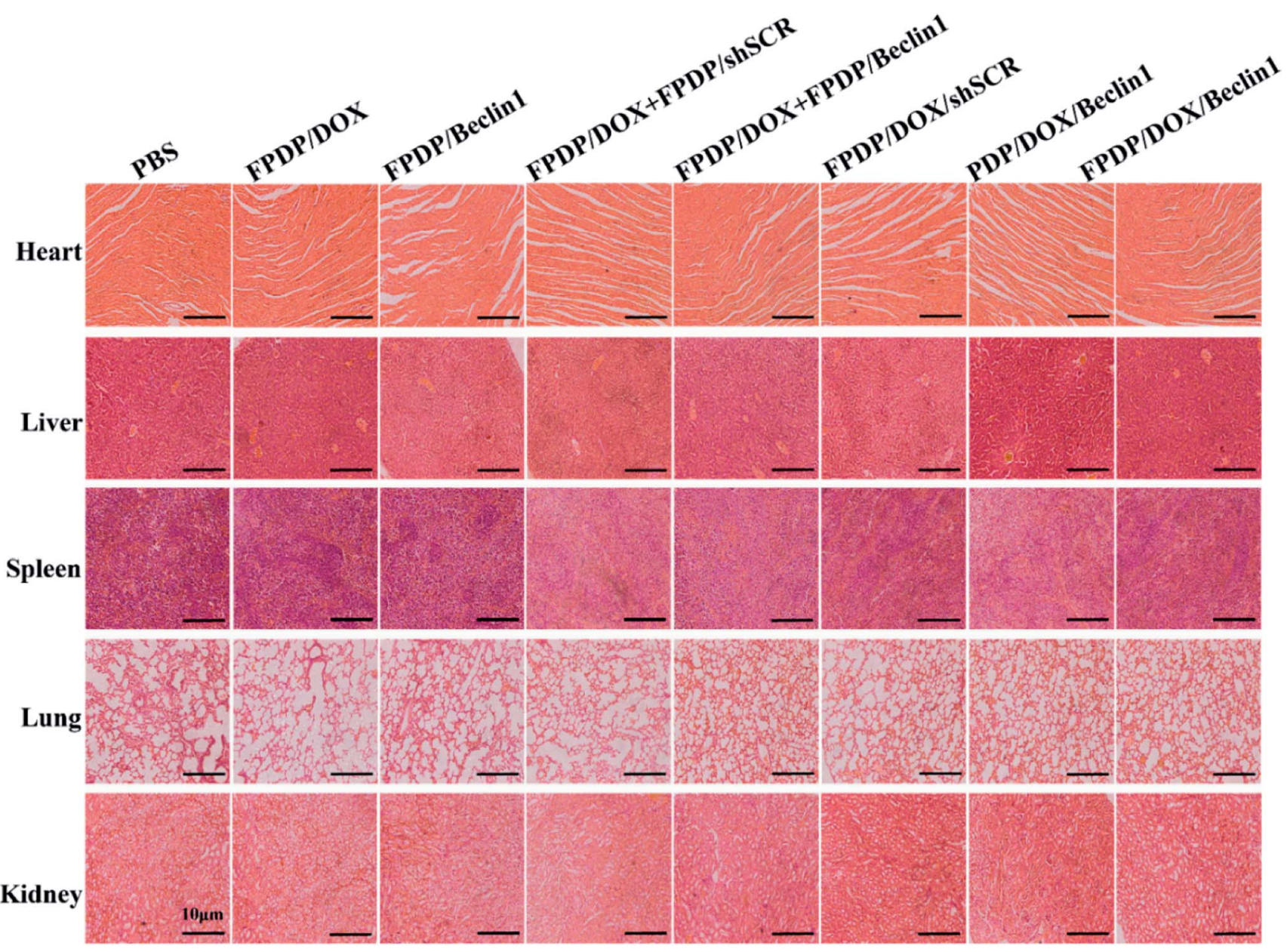

Fig. 6 Histologic assessments of major organs with HE staining in mice. 
Free DOX

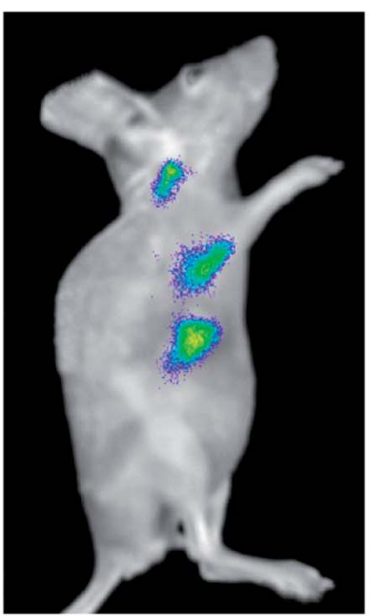

FPDP/DOX

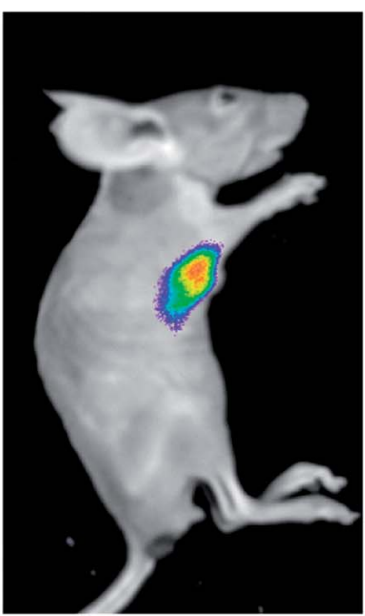

PDP/DOX/shBeclin1 FPDP/DOX/shBeclin1

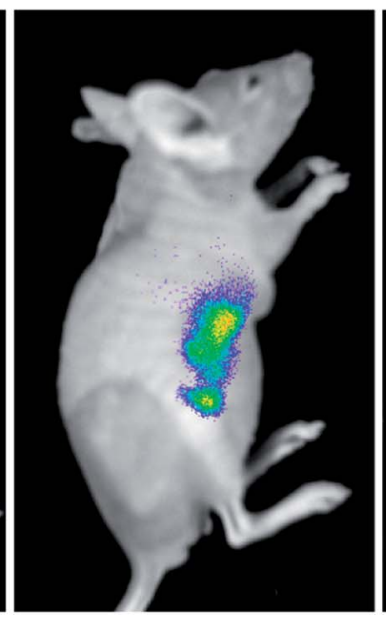

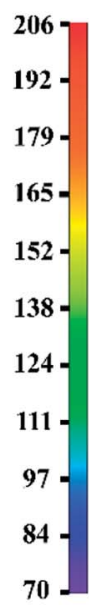

Fig. 7 In vivo bioimaging of DOX delivered by FPDP nano-complexes or its reference formulations injected into tumor-bearing nude mice monitored by an NIR fluorescence imaging system.

in both FPDP/DOX and FPDP/DOX/shBeclin1 groups, the red fluorescence signal of DOX centralized in tumor was stronger in comparison with that in PDP/DOX/shBeclin1 groups, demonstrating the excellent tumor targeting ability of FPDP micelles and the FA in FPDP micelles enhanced the uptake efficiency of FPDP/DOX and FPDP/DOX/shBeclin1 through FR-mediated endocytosis. This result was consistent with the higher cytotoxicity of FPDP/DOX/shBeclin1 in vitro and antitumor efficacy in vivo.

\section{Conclusions}

In summary, we synthesized a novel FR-targeted copolymer FPDP for effective targeted co-delivery of DOX and shBeclin1 for improving the anticancer effect of DOX through the blockade of autophagy process for the first time. FPDP exhibited good biocompatibility and excellent loading capacities for both DOX and shBeclin1. Meanwhile, FPDP/DOX micelles could effectively target $\mathrm{FR}^{+}$cancer cells via the $\mathrm{FR}$ mediated endocytosis process. Most importantly, the FPDP micelles could efficiently co-deliver shBeclin1 and DOX into the cytoplasm of the same HeLa cells, exhibiting the strongest synergistic antitumor efficacy compared with FPDP/DOX micelles or FPDP/shBeclin1 alone. Excitedly, in HeLa-bearing nude mice, FR-targeted FPDP/DOX/ shBeclin1 could be delivered to the tumor site, and suppressed cancer growth more effectively than delivery of either DOX or shBeclin1 separately in cancer therapy. This study indicated the great potential of the FPDP to serve as an effective carrier for enhancing the intracellular co-delivery of DOX and therapeutic shBeclin1 in cancer cells overexpressing FR to improve the anticancer effect of DOX through the blockade of autophagy process in cancer therapy.

\section{Conflicts of interest}

There are no conflicts to declare.

\section{Acknowledgements}

This research was financially supported by the National Natural Science Foundation of China (Grant Number. 21376039; U1608222).

\section{References}

1 H. T. Ta, C. R. Dass, I. Larson, P. F. Choong and D. E. Dunstan, A chitosan-dipotassium orthophosphate hydrogel for the delivery of Doxorubicin in the treatment of osteosarcoma, Biomaterials, 2009, 30, 3605-3613.

2 G. Minotti, P. Menna, E. Salvatorelli, G. Cairo and L. Gianni, Anthracyclines: molecular advances and pharmacologic developments in antitumor activity and cardiotoxicity, Pharmacol. Rev., 2004, 56, 185-229.

3 R. I. Pakunlu, Y. Wang, W. Tsao, V. Pozharov, T. J. Cook and T. Minko, Enhancement of the Efficacy of Chemotherapy for Lung Cancer by Simultaneous Suppression of Multidrug Resistance and Antiapoptotic Cellular Defense: Novel Multicomponent Delivery System, Cancer Res., 2004, 64, 6214-6224.

4 A. Kuma, M. Hatano, M. Matsui, A. Yamamoto and H. Nakaya, The role of autophagy during the early neonatal starvation period, Nature, 2004, 432, 1032-1036.

5 J. C. Farre, R. Krick, S. Subramani and M. Thumm, Turnover of organelles by autophagy in yeast, Curr. Opin. Cell Biol., 2009, 21, 522-530.

6 Y. L. Hu, A. Jahangiri, M. Delay and M. K. Aghi, Tumor cell autophagy as an adaptive response mediating resistance to treatments such as antiangiogenic therapy, Cancer Res., 2012, 72, 4294-4299.

7 A. Herman-Antosiewicz, D. E. Johnson and S. V. Singh, Sulforaphane causes autophagy to inhibit release of cytochrome $\mathrm{C}$ and apoptosis in human prostate cancer cells, Cancer Res., 2006, 66, 5828-5835. 
8 B. Levine and G. Kroemer, Autophagy in the pathogenesis of disease, Cell, 2008, 132, 27-42.

9 R. Kang, H. J. Zeh, M. T. Lotze and D. Tang, The Beclin 1 network regulates autophagy and apoptosis, Cell Death Differ., 2011, 18, 571-580.

10 R. C. Wang, Y. J. Wei, Z. Y. An, Z. J. Zou, G. H. Xiao, G. Bhagat, M. White, J. Reichelt and B. Levine, Aktmediated regulation of autophagy and tumorigenesis through Beclin 1 phosphorylation, Science, 2012, 338, 956959.

11 A. M. Butt, M. C. I. M. Amin, H. Katas, N. A. A. Murad, R. Jamal and P. Kesharwani, Doxorubicin and siRNA Codelivery via Chitosan-Coated pH-Responsive Mixed Micellar Polyplexes for Enhanced Cancer Therapy in Multidrug-Resistant Tumors, Mol. Pharm., 2016, 13, 41794190.

12 Q. Chen, M. Xu, W. Zheng, T. Xu, H. Deng and J. Liu, Se/RuDecorated Porous Metal-Organic Framework Nanoparticles for The Delivery of Pooled siRNAs to Reversing Multidrug Resistance in Taxol-Resistant Breast Cancer Cells, ACS Appl. Mater. Interfaces, 2017, 9, 6712-6724.

13 X. J. Zhou, L. Chen, W. Nie, W. Z. Wang, M. Qin, X. M. Mo, H. S. Wang and C. L. He, Dual-Responsive Mesoporous Silica Nanoparticles Mediated Codelivery of Doxorubicin and Bcl-2 SiRNA for Targeted Treatment of Breast Cancer, J. Phys. Chem. C, 2016, 120, 22375-22387.

14 K. Y. Choi, O. F. Silvestre, X. L. Huang, K. H. Min, G. P. Howard, N. Hida, A. J. Jin, N. Carvajal, S. W. Lee, J.-In. Hong and X. Y. Chen, Versatile RNA Interference Nanoplatform for Systemic Delivery of RNAs, ACS Nano, 2014, 8, 4559-4570.

15 F. Daniel, A. Legrand, D. Pessayre, N. Vadrot, V. Descatoire and D. Bernuau, Partial Beclin 1 silencing aggravates doxorubicin- and Fas-induced apoptosis in HepG2 cells, World J. Gastroenterol., 2006, 12, 2895-2900.

16 J. Li, Y. Wang, Y. Zhu and D. Oupicky, Recent advances in delivery of drug-nucleic acid combinations for cancer treatment, J. Controlled Release, 2013, 172, 589-600.

17 S. Y. Lee, C. Y. Yang, C. L. Peng, M. F. Wei, K. C. Chen, C. J. Yao and M. J. Shieh, A theranostic micelleplex codelivering SN-38 and VEGF siRNA for colorectal cancer therapy, Biomaterials, 2016, 86, 92-105.

18 J. Jiang, S. J. Yang, J. C. Wang, L. J. Yang, Z. Z. Xu, T. Yang, X. Y. Liu and Q. Zhang, Sequential treatment of drugresistant tumors with RGD-modified liposomes containing siRNA or doxorubicin, Eur. J. Pharm. Biopharm., 2010, 76, 170-178.

19 T. L. Kaneshiro and Z. R. Lu, Targeted intracellular codelivery of chemotherapeutics and nucleic acid with a well-defined dendrimer-based nanoglobular carrier, Biomaterials, 2009, 30, 5660-5666.

20 T. T. Li, X. Shen, Y. Geng, Z. Y. Chen, L. Li, S. Li, H. Yang, C. H. Wu, H. J. Zeng and Y. Y. Liu, Folate-Functionalized Magnetic-Mesoporous Silica Nanoparticles for Drug/Gene Codelivery To Potentiate the Antitumor Efficacy, ACS Appl. Mater. Interfaces, 2016, 8, 13748-13758.
21 Z. Z. Chen, P. P. Zhu, Y. S. Zhang, Y. T. Liu, Y. L. He, L. F. Zhang and Y. F. Gao, Enhanced Sensitivity of Cancer Stem Cells to Chemotherapy Using Functionalized Mesoporous Silica Nanoparticles, Mol. Pharm., 2016, 13, 2749-2759.

22 J. M. Li, Y. Y. Wang, M. X. Zhao, C. P. Tan, Y. Q. Li, X. Y. Le, L. N. Ji and Z. W. Mao, Multifunctional QD-based co-delivery of siRNA and doxorubicin to HeLa cells for reversal of multidrug resistance and real-time tracking, Biomaterials, 2012, 33, 2780-2790.

23 T. J. Yin, L. Wang, L. F. Yin, J. P. Zhou and M. R. Huo, Codelivery of hydrophobic paclitaxel and hydrophilic AURKA specific siRNA by redox-sensitive micelles for effective treatment of breast cancer, Biomaterials, 2015, 61, 10-25.

24 C. F. Zheng, M. B. Zheng, P. Gong, J. Z. Deng, H. Q. Yi, P. F. Zhang, Y. J. Zhang, P. Liu, Y. F. Ma and L. T. Cai, Polypeptide cationic micelles mediated co-delivery of docetaxel and siRNA for synergistic tumor therapy, Biomaterials, 2013, 34, 3431-3438.

25 D. Cheng, N. Cao, J. F. Chen, X. S. Yu and X. T. Shuai, Multifunctional nanocarrier mediated co-delivery of doxorubicin and siRNA for synergistic enhancement of glioma apoptosis in rat, Biomaterials, 2012, 33, 1170-1179.

26 S. P. Wang, J. M. Zhang, Y. T. Wang and M. W. Chen, Hyaluronic acid-coated PEI-PLGA nanoparticles mediated co-delivery of doxorubicin and miR-542-3p for triple negative breast cancer therapy, Nanomedicine, 2016, 12, 411-420.

27 Y. Y. Liu, Y. Wang, C. Zhang, P. Zhou, Y. Liu, T. An, D. X. Sun, N. Zhang and Y.S. Wang, Core-shell nanoparticles based on pullulan and poly(beta-amino) ester for hepatoma-targeted codelivery of gene and chemotherapy agent, ACS Appl. Mater. Interfaces, 2014, 6, 18712-18720.

28 R. S. Singh, N. Kaur and J. F. Kennedy, Pullulan and pullulan derivatives as promising biomolecules for drug and gene targeting, Carbohydr. Polym., 2015, 123, 190-207.

29 H. N. Li, Y. Sun, J. Liang, Y. Fan and X. Zhang, pH-Sensitive pullulan-DOX conjugate nanoparticles for co-loading PDTC to suppress growth and chemoresistance of hepatocellular carcinoma, J. Mater. Chem. B, 2015, 3, 8070-8078.

30 L. L. Chen, F. L. Ji, Y. M. Bao, J. Xia, L. Y. Guo, J. Y. Wang and Y. C. Li, Biocompatible cationic pullulan-g-desoxycholic acid-g-PEI micelles used to co-deliver drug and gene for cancer therapy, Mater. Sci. Eng. C., 2017, 70, 418-429.

$31 \mathrm{Y}$. J. $\mathrm{Lu}$ and P. S. Low, Folate-mediated delivery of macromolecular anticancer therapeutic agents, Adv. Adv. Drug Delivery Rev., 2012, 64, 342-352.

32 X. Wang, Y. Q. Wang, J. Li, S. Nannapaneni, M. Tighiouart, Z. Chen, S. M. Nie and D. Shin, A Folate ReceptorTargeting Nanoparticle Minimizes Drug Resistance in a Human Cancer Model, ACS Nano, 2011, 5, 6184-6194.

33 J. M. Qian, M. H. Xu, A. L. Suo, W. J. Xu, T. Liu, X. F. Liu, Y. Yao and H. J. Wang, Folate-decorated hydrophilic threearm star-block terpolymer as a novel nanovehicle for targeted co-delivery of doxorubicin and Bcl-2 siRNA in breast cancer therapy, Acta Biomater., 2015, 15, 102-116. 
34 S. Chen, L. Rong, Q. Lei, P. X. Cao, S. Y. Qin, D. W. Zheng, H. Z. Jia, J. Y. Zhu, S. X. Cheng, R. X. Zhuo and X. Z. Zhang, A surface charge-switchable and folate modified system for co-delivery of proapoptosis peptide and p53 plasmid in cancer therapy, Biomaterials, 2016, 77, 149-163.

35 J. Y. Wang, B. R. Dou and Y. M. Bao, Efficient targeted pDNA/ siRNA delivery with folate-low-molecular-weight polyethyleneimine-modified pullulan as non-viral carrier, Mater. Sci. Eng. C., 2014, 34, 98-109.

36 I. R. Vlahov and C. P. Leamon, Engineering folate-drug conjugates to target cancer: from chemistry to clinic, Bioconjugate Chem., 2012, 23, 1357-1369.

37 Y. F. Wang, P. F. Liu, Y. R. Duan, X. Yin, Q. Wang, X. F. Liu, X. R. Wang, J. H. Zhou, W. W. Wang, L. H. Qiu and W. Di, Specific cell targeting with APRPG conjugated PEG-PLGA nanoparticles for treating ovarian cancer, Biomaterials, 2014, 35, 983-992.

38 M. Q. Li, Z. H. Tang, S. X. Lv, W. T. Song, H. Hong, X. B. Jing, Y. Y. Zhang and X. S. Chen, Cisplatin crosslinked $\mathrm{pH}-$ sensitive nanoparticles for efficient delivery of doxorubicin, Biomaterials, 2014, 35, 3851-3864.

39 X. C. Yang, Y. L. Niu, N. N. Zhao, C. Mao and F. J. Xu, A biocleavable pullulan-based vector via ATRP for liver celltargeting gene delivery, Biomaterials, 2014, 35, 3873-3884.

40 X. W. Wang, J. Y. Wang, Y. M. Bao, B. H. Wang, X. H. Wang and L. L. Chen, Novel reduction-sensitive pullulan-based micelles with good hemocompatibility for efficient intracellular doxorubicin delivery, $R S C A d v$., 2014, 4, 60064-60074.
41 C. L. Fu, L. Lin, H. L. Shi, D. X. Zheng, W. Wang, S. Q. Gao, Y. F. Zhao, H. Y. Tian, X. J. Zhu and X. S. Chen, Hydrophobic poly (amino acid) modified PEI mediated delivery of revcasp-3 for cancer therapy, Biomaterials, 2012, 33, 4589-4596.

42 S. N. Zhao, L. V. Minh, N. Li, V. M. Garamus, U. A. Handge, J. W. Liu, R. G. Zhang, R. Willumeit-Römer and A. Zou, Doxorubicin hydrochloride-oleic acid conjugate loaded nanostructured lipid carriers for tumor specific drug release, Colloids Surf., B, 2016, 145, 95-103.

43 K. Na, K. H. Park, S. W. Kim and Y. H. Bae, Self-assembled hydrogel nanoparticles from curdlan derivatives: characterization, anti-cancer drug release and interaction with a hepatoma cell line (HepG2), J. Controlled Release, 2000, 69, 225-239.

44 X. W. Guan, Z. P. Guo, L. Lin, J. Chen, H. Y. Tian and X. S. Chen, Ultrasensitive $\mathrm{pH}$ Triggered Charge/Size DualRebound Gene Delivery System, Nano Lett., 2016, 16, 68236831.

45 H. Z. Jia, W. Zhang, J. Y. Zhu, B. Yang, S. Chen, G. Chen, Y. F. Zhao, J. Feng and X. Z. Zhang, Hyperbranchedhyperbranched polymeric nanoassembly to mediate controllable co-delivery of siRNA and drug for synergistic tumor therapy, J. Controlled Release, 2015, 216, 9-17.

46 S. Y. Lee, M. S. Huh, S. Lee, S. J. Lee, H. Chung, J. H. Park, Y. K. Oh, K. Choi, K. Kim and I. C. Kwon, Stability and cellular uptake of polymerized siRNA (poly-siRNA)/ polyethylenimine (PEI) complexes for efficient gene silencing, J. Controlled Release, 2010, 141, 339-346. 